International Journal of Engineering \& Technology, $7(2.21)(2018) 1-4$
International Journal of Engineering \& Technology
WPC
Website: www.sciencepubco.com/index.php/IJET
Research paper

\title{
Design and development of a novel triphibian quadcopter
}

\author{
T. Prajwal Shenoy ${ }^{*}$, K. Praveen Shenoy ${ }^{2}$, Lukhman Khan ${ }^{3}$, Sabdar Aziz ${ }^{4}$, Sayed Afran ${ }^{5}$, Kamlesh Kumar $^{6}$ \\ ${ }^{I}$ Assistant Professor, Department of Mechatronics Engineering, Manipal Institute of Technology, Manipal. \\ ${ }^{2}$ Department of Mechatronics Engineering, Manipal Institute of Technology, Manipal University, Manipal. \\ ${ }^{3}$ Department of Mechatronics Engineering, Manipal Institute of Technology, Manipal University, Manipal. \\ ${ }^{4}$ Department of Mechatronics Engineering, Manipal Institute of Technology, Manipal University, Manipal. \\ ${ }^{5}$ Department of Mechatronics Engineering, Manipal Institute of Technology, Manipal University, Manipal. \\ ${ }^{6}$ Assistant Professor (Sr scale), Department of Aeronautical and Automobile Engineering, Manipal Institute of Technology.
}

\begin{abstract}
Multimodal Vehicles can travel in at least two distinctive modes of transportation, be it air and water, land and water or air and land. In this paper, design and development of a novel unmanned multimodal vehicle is presented that can travel in air, land and water, also called a as a triphibian quadcopter which would prove beneficial in rescue operations and operations involving hazardous environments. The vehicle is essentially a mix of unmanned aerial vehicle, unmanned ground vehicle and unmanned surface vehicle and is equipped with mechanisms to travel in land, air and water at the will of the operator. The entire framework of the vehicle is built on the multi rotor configuration. Flight tests were conducted to validate the design.
\end{abstract}

Keywords:Multimodal, triphibian, UAV, UGV, USV.

\section{Introduction}

Multimodal vehicles that travel in all 3 modes viz. land air and water find itself useful in several applications like search and rescue operations where the vehicle might need to hover in congested areas without lot of room for flight. In such situations, the vehicle can travel in land or water and carry out the required rescue operation. Such vehicles can also be used in land mine detection where the vehicle travels in land for detection rather than hovering in air, thus vastly saving the battery life of the vehicle. But design of such a system is usually complicated considering the weight to be lifted, control of flight and battery life.

For any vehicle to fly, there are two basic forms of aircraft: fixed wing and rotary wing aircraft. A fixed wing like any commercial aero plane has a fuselage, which by means of burning fuel produces thrust to move forward and take off. On the other hand, a rotary wing aircraft like a helicopter can gain lift by rotating blades without the requirement of a forward speed. Since a rotary winged aircraft can gain lift without getting a forward speed.Such a vehicle does not need a runway or a track, since it requires only a small area for landing and takeoff. Rotary wing aircrafts have been widely used in emergency needs in places that have no runways/tracks or cannot have tracks. Due to their characteristic of flying in low speed or even staying still in air, rotary wing aircrafts are also good tools for measuring, surveying, detecting, and rescuing operations.

A few approaches for multimodal vehicles were reviewed from past literature. A micro triphibian quad copter can be configured with wheels provided to traverse on land, four rotors for travelling in air and by an additional mechanism to float in water [1]. The wheels are extremely light weight and provide the necessary buoyancy when in water. The rotation of the wheels in water causes the vehicle to move forward. The design makes use of pulleys and belts to transmit power to the wheels from motors mounted centrally thus reducing weight. The triphibian quad copter could also have a special set of wheels to traverse on land which can also climb through terrains [2]. If the size of the quad copter is small, then it can travel and recover on terrains even after a 180-degree flip. These quad copters can also be used for photography as it can land in water and is extremely water proof [3].

Even though available literature on multimodal quad copters are scarce, many patented designs on manned multimodal vehicles have been reported in the past. A vehicle for military purpose can be developed, which could be a normal four wheeled manned vehicle consisting of an air parachute which is deployable, and a source of propulsion for flying, like a vertical ducted fan or propeller requiring a forward speed for takeoff [4]. Wheeled vehicles provided with rotary blades that can be tilted in vertical direction [5] is beneficial as opposed to horizontal blades in a helicopter. The blades can be tilted either to obtain lift or forward speed and can also be operated in water. A convertible passenger vehicle [6] would revolutionize transportation as it can traverse in land, air and water. It could have wings attached to a propelling device which can be tilted to provide thrust in any direction with a provision for tail assembly for maneuvering in land and water. The wings could be designed in such a way that it can retracted and stored in the main body when moving on road.

From the literature, it can be observed that few of the existing multimodal unmanned quad copters are micro and light weight and not meant for performing actions like lifting heavy weights. Manned multimodal vehicles are mostly fixed wing aircrafts capable of only traversing in the 3 modes but does not perform any task. In this paper, design and development of a heavy duty multimodal quad copter is discussed which is capable of traversing in air, land and water by utilizing the appropriate drive mechanism depending on the conditions at the will of the operator and can also be further customized to perform heavy duty applications like lifting of weights. The vehicle uses multi rotor drive system in Unmanned Aerial Vehicle (UAV) mode, a four- 
wheel geared-motor drive system in Unmanned Ground Vehicle (UGV) mode and a customized blade mechanism in Unmanned Surface Vehicle (USV) mode. Basic configuration and principle of vehicle in UAV mode in analogous to that of a quad copter. The four-wheel drive propagation mechanism driven by four geared motors (two motors on either side of the vehicle) in UGV mode gives vehicle an added benefit of providing the extra torque and superior control. The USV mode of the vehicle is driven by customized steel plates that provide enough thrust to cut through the water resistance.

\section{Methodology}

To design the multimodal vehicular system, the mechanical frame work is modeled using CAD. Calculations are done for floatation and selection of electronic components depending on the load to be lifted. The prototype of the mechanical model is created and the final assembled system is subjected to flight testing. Details of modeling, electronic component selection and prototyping is discussed in the subsequent sections.

\section{Mechanical modeling of UAV and UGV frames}

The vehicle is divided into two sub- assemblies namely UAV assembly and UGV/USV assembly. Both the units are then assembled to form the final multimodal quad copter. The description of UAV and UGV subassemblies are discussed in next sections.

\section{UAV frame design}

An aluminum frame is considered for rigidity purpose. The frame of the system is modeled using approximate dimensions with a hollow cross section of $1.9 \mathrm{~cm} \mathrm{x} 1.9 \mathrm{~cm}$ and stretching out up to a

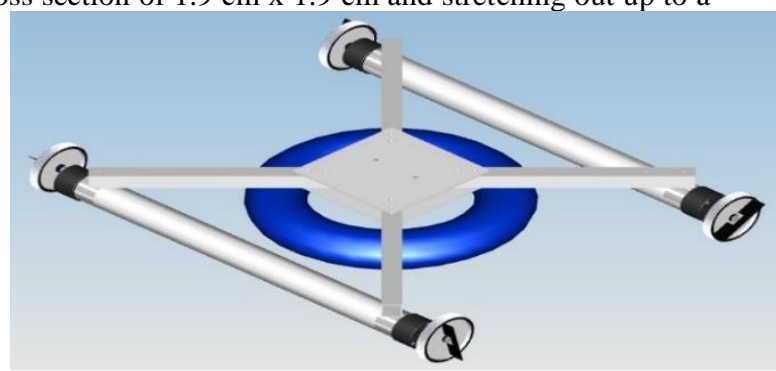

Fig. 2: CAD assembly of multimodal vehicle

\section{Selection of electronic components for UAV, UGV and USV}

\section{UAV sub-assembly}

The motors are required to carry a load of approximately $1.4 \mathrm{~kg}$ (plus additional load of electronics and the battery). Therefore, motors with low $\mathrm{kV}$ rating are required which have higher load carrying capacity. Hence Turnigy $920 \mathrm{kV}$ motors are chosen. Standard 3-S Lithium-Polymer battery is used to supply a voltage of $11.1 \mathrm{~V}$. Since the $\mathrm{kV}$ ratings refer to RPM per Voltage, the motors would spin at approximately 10,200 RPM at no load. Each motor has a thrust rating of 800 grams, hence four motors would provide a thrust of 3,200 grams which is sufficient to propel the vehicle in air. The motors have a maximum current rating of 20 Amperes which refers to the maximum current that the motor can draw. Hence Electronic speed controllers (ESC's) are chosen with current rating of 30 Amperes just for the matter of safety. As the motors draw a maximum current of 20 Amperes and the battery would supply a voltage of $11.1 \mathrm{~V}$, the power in Watt would be equal to VI = $222 \mathrm{Watt}$. As each motor draws a maximum current length of $21 \mathrm{~cm}$ with a thickness of $0.1 \mathrm{~cm}$. The aluminum profiles are connected to the plywood base plate in cross formation. The motor mounts are attached towards the end of the profile. The total weight of UAV frame is observed to be approximately 750 grams. The CAD model of the frame is shown in Fig1.

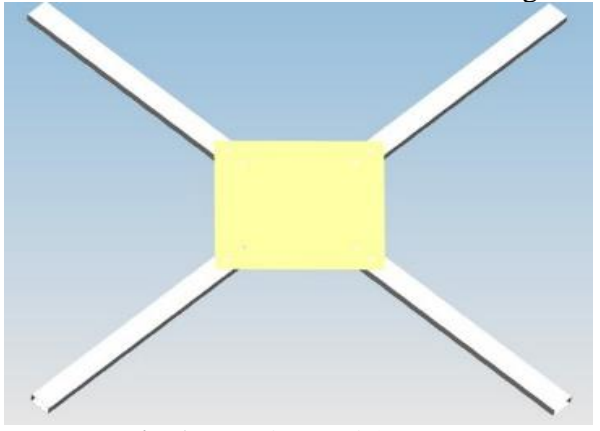

Fig. 1: Base frame of the UAV

\section{UGV and USV frame design}

UGV frame is designed to be circular and hollow PVC pipe of diameter $3.81 \mathrm{~cm}$ and has a length of $39 \mathrm{~cm}$ to suit the frame of the UAV. The circular shape enables the sealing of the motors in the pipe during its navigation in water. The pipes also enable better stability and buoyancy when in water. Instead of using separate propeller mechanism to drive the vehicle in water, a flat metal piece made from stainless steel is attached to the central axis of each wheel. For floatation purpose, a tube filled with air is used. The CAD assembly of the vehicle is shown in Fig 2. The calculation for flotation is discussed later after the selection of electronic components. The total weight of the components to be used to develop CAD assembly into a prototype was observed to be $1.4 \mathrm{~kg}$. The conceptual design of the vehicle after addition of all the electronic components is shown in Fig 3.

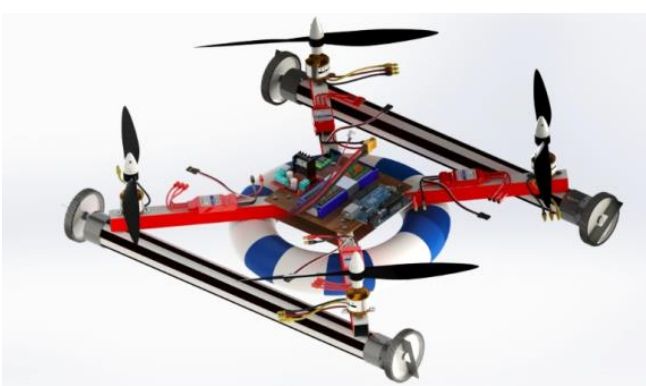

Fig. 3: Complete assembly of multimodal vehicle

of 20 Amperes, at least 80 Amperes is required at full load. Hence Turnigy 4,000 mAh Li-Po battery with $30 \mathrm{C}$ discharge ratings was chosen which could supply a maximum current of 120 Amperes (maximum current from battery is equivalent to capacity of battery multiplied with discharge ratings). The BrainFPV RE1 is chosen as flight controller because of its advanced features such as builtin graphical OSD, fast processor (STM32 F3, 180MHz), lap timing support (Trackmate, I-lap, configurable using OSD menu), programmable LED support and telemetry. The flight controller would directly stack on top of the BrainFPVmPB (mini power board) which would distribute the power to all the electrical components.

FlySky FS-i6 radio system, which works at frequency of $2.4 \mathrm{GHz}$ is used to remotely operate the vehicle. The radio system has 6 channel functions, sensitive receiver chip, and $500 \mathrm{kHz}$ bandwidth and uses low power electronic components to reduce power consumption.

\section{UGV and USV sub-assembly}

After the addition of electronic components of UAV sub-assembly to the framework, the vehicle would weigh around $1.9 \mathrm{~kg}$, so the 
motors that would be used to drive the vehicle on the ground will have to bear the load acting on it. Geared DC motors which has rated speed of 100RPM and equivalent torque of $1.5 \mathrm{~kg}-\mathrm{cm}$ are chosen. As four DC motors are used, the torque delivered to the wheels was $6 \mathrm{~kg}-\mathrm{cm}$ which is sufficient to drive the vehicle at expected speed. The USV didn't require any electrical components as it is the mechanical modification of UGV assembly. After addition of DC geared motors, customized steel plates and its constituent electrical components, the vehicle would weigh around $2.28 \mathrm{Kg}$.

\section{Mathematical calculations for floatation}

The vehicle must float in water with all the load acting on it and also has to propel in water. Circular air-filled tube of 12 inch is used as floating mechanism to prevent the vehicle from sinking in water. The tube is attached to the vehicle in such a way that the center of gravity of the vehicle will coincide with the center of the tube so that the vehicle will have good stability while travelling in water. The mathematical deductions of the circular air-filled tube relating to pressure, density and buoyant forces are discussed. The properties of the tube are shown in Table 1.

Table 1: Parameters of Circular Tube

\begin{tabular}{|l|l|}
\hline Parameter & Value \\
\hline Air pressure in the circular tube & 8 bar \\
\hline External diameter of the tube & 12 inch $(30.48 \mathrm{~cm})$ \\
\hline Cross section diameter of the tube & 2.2 inch $(5.60 \mathrm{~cm})$ \\
\hline Circumference of the tube & $95.72 \mathrm{~cm}$. \\
\hline Cross sectional area of tube & $24.61 \mathrm{~cm}^{2}$ \\
\hline $\begin{array}{l}\text { Volume of air filled in circular tube = Area } \mathrm{x} \\
\text { circumference }\end{array}$ & $2,356 \mathrm{~cm}^{3}$ \\
\hline
\end{tabular}

From Ideal gas law

$\mathrm{PV}=\mathrm{mRT}$

Where $\mathrm{P}$ is pressure, $\mathrm{V}$ is volume, $\mathrm{m}$ is the mass, $\mathrm{T}$ is temperature and $\mathrm{R}$ is universal gas constant. Since density $\rho$ can be written as $\mathrm{m} / \mathrm{V}$,

$\rho=\frac{P}{R T}$

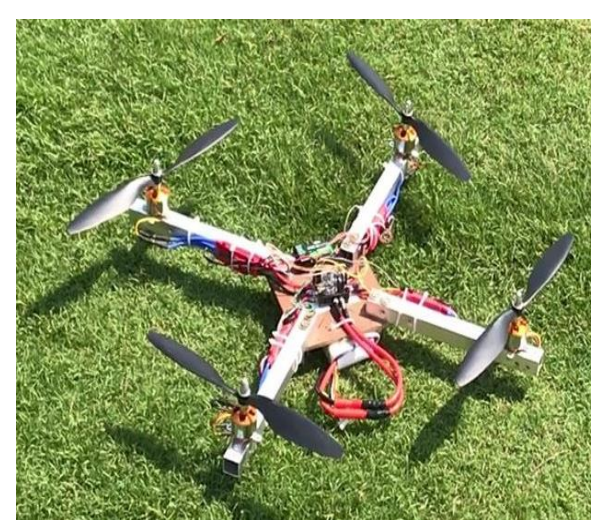

Fig. 4: UAV sub-assembly

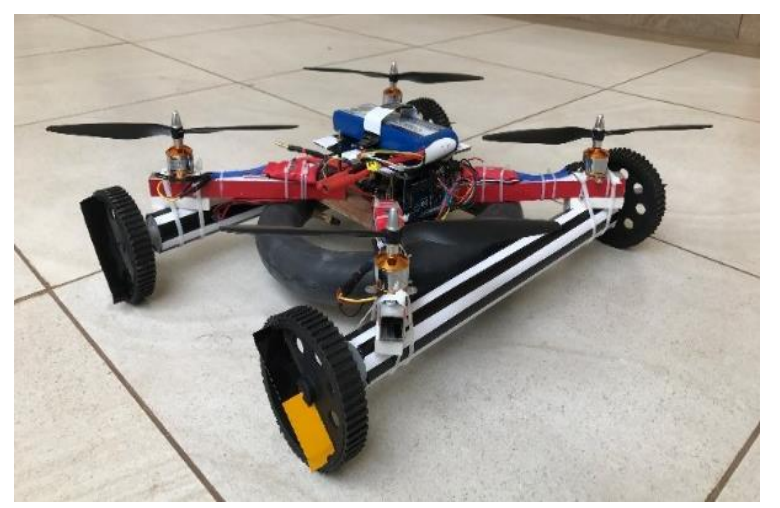

Fig. 6: Unmanned multimodal vehicle
By substituting $\mathrm{T}=300 \mathrm{~K}, \mathrm{R}=287 \mathrm{~J} / \mathrm{kgK}$ and $\mathrm{P}=8$ bar in above equation, we get $\rho=9.286 \mathrm{~kg} / \mathrm{m}^{3}$. From concepts of buoyancy,

Weight of the vehicle $=$ weight of the water displaced

Weight of the vehicle $=2.28 \mathrm{~kg}(21 \mathrm{~N})$

Weight of water displaced $=$ Weight density $\mathrm{x}$ Volume of vehicle submerged

By equating (3), (4) and (5), the extent of depth up to which the air-filled tube would submerge in water can be found

$21 \mathrm{~N}=1000 \times 9.81 \times \pi \mathrm{r}^{2} \times \mathrm{h}$

$\mathrm{h}=0.02 \mathrm{~m}$

Hence approximately $2 \mathrm{~cm}$ of the circular tube would be submerged while propelling in water while approximately $2.4 \mathrm{~cm}$ of the tube would be above the surface of water. This theoretical depth of immersion is in safe limits and gives conclusive proof to proceed with the development of prototype.

\section{Development of prototype}

The mechanical components that were discussed in previous sections are customized and assembled to suite the requirement. The structural framework of the vehicle is prepared. The mechanical sub-assemblies are developed and tested for their structural stability. Electrical components are programmed and attached to sub-assemblies to complete the process of developing the prototype. The completed UAV sub assembly is shown in Fig 4 .

The electrical system is considered most critical for the development of a stable prototype. All parts and sub-assemblies are integrated together to form the final assembly of unmanned multimodal vehicle. The weight of the vehicle is measured and it agreed with the calculations discussed in previous sections. The final assembly of unmanned multimodal vehicle is shown in Fig 5 and Fig 6.

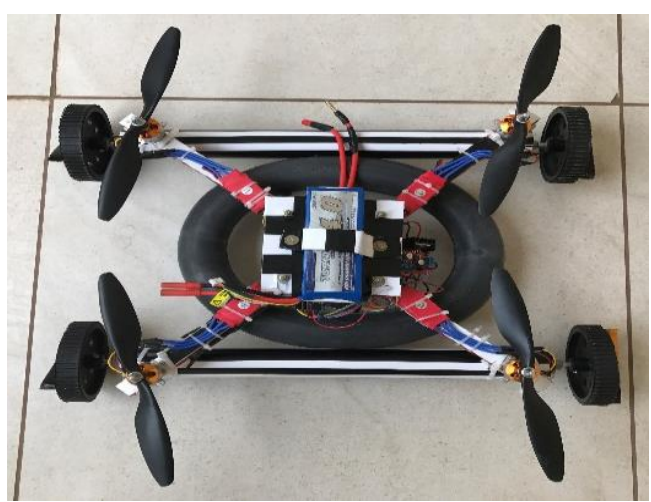

Fig. 5: Unmanned multimodal vehicle-Top View

\section{Performance tests and results}

The prototype was tested for capabilities in air, land and water. While in air, the vehicle attained a maximum controllable height of around 15 meters and recorded a flight time of around 7 minutes. The flight was extremely stable and can be further customized to perform additional tasks like lifting heavy weights or perform tasks in ground like land mine detection. The multimodal vehicle photographed while in air,3 storeys high, is shown in Fig 7. While travelling in land, the vehicle was capable of moving forward, backward and taking left or right turn at the will of the operator. The motors of the vehicle were capable of bearing the weight of the vehicle without affecting the RPM. 
The vehicle was tested for its performance in water in a pool. The vehicle was able to propagate in water at moderate speed and the blade mechanism was able to cut through the water to propel the vehicle. The vehicle was also able to land and take off from the water with extreme stability. The mathematical calculation carried out to determine the depth of immersion of fully inflated circular

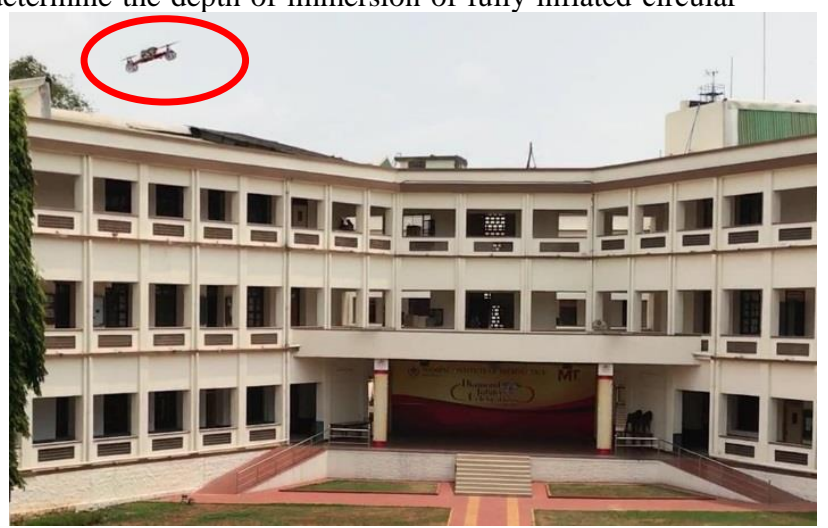

Fig. 7: Unmanned multimodal vehicle - flight test tube was in agreement with the experimental results. The circular tube sank up to a depth of $2.3 \mathrm{~cm}$ and also balanced the weight of the vehicle. The multimodal vehicle travelling in water is shown in Fig 8.

\section{Conclusion}

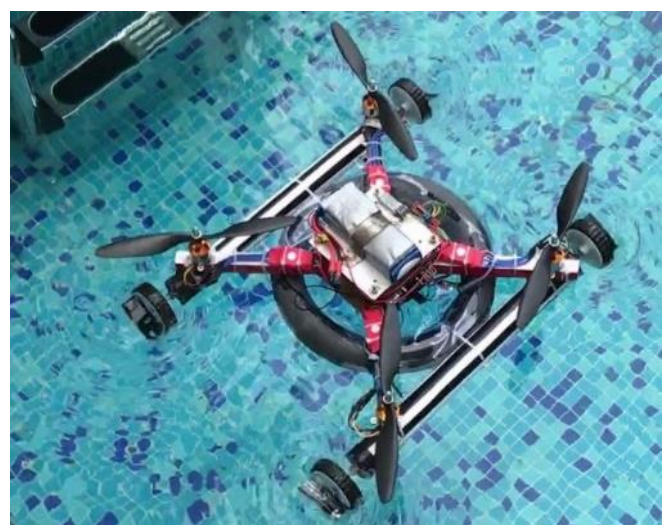

Fig. 8: Multimodal vehicle propelling in water

The novel triphibian quadcopter has been successfully designed and developed and can be used for several applications especially those where human intervention is not feasible. The triphibianquad copter can be easily used in UGV modes for ground applications instead of hovering for a long time at low heights resulting in increased battery life. Further attachments can be added to make the vehicle perform actions for required applications like additional retractable robotic arm for bomb disposal or material handling in nuclear reactors.

\section{Acknowledgement}

The above mentioned prototype is a part of graduate project developed under the guidance and immense support from the Faculty of Department of Mechatronics Engineering, Manipal University and Centre for Avionics, Manipal University. The above mentioned prototype has been filed for intellectual property rights with Indian patent application number: 201741027807 dated $4^{\text {th }}$ August 2017.

\section{References}

[1] Hazel W, "Micro triphibian quadcopter", US Patent 201510306015, (2015).

[2] Tao S \& Yimin S, "Triphibian wheel-like mobile robot", CN103522855A, (2014).

[3] Bradlow HW \& Balaresque A, "Launching unmanned aerial copter from mid-air", US20160179096 A1, (2016).

[4] Preston D, "Multimodal, deployable vehicle", U.S.Patent 7066426 $B 2,(2006)$.

[5] Quanwen, Guowen \& Wanbing, "Vehicle capable of travelling in land, air or water, World Intellectual Property Organisation" PCT/CN2009/074357, (2009).

[6] Seiford DS, "Convertible vehicle for road, air and water usage", U.S.Patent WO 2011066004 A3, (2009).

[7] Manarvi IA, Aqib M, Ajmal M, Usman M, Khurshid S \& Sikandar $\mathrm{U}$, "Design and development of a quad copter (UMAASK) using CAD/CAM/CAE", IEEE Aerospace Conference, (2013), pp.1-10.

[8] Mahen MA, Anirudh S, Naik A, Chethana HD \& Shashank AC, "Design and development of amphibious quadcopter", International Journal of Mechanical and Production Engineering, Vol.2, No.7, (2014), pp.30-34.

[9] Yang X, Wang, Liang J, Yao G \& Liu M, "Survey on the novel hybrid aquatic-aerial amphibious aircraft: Aquatic unmanned aerial vehicle (AquaUAV)", Progress in Aerospace Sciences, Vol.74, (2015), pp.131-151.

[10] Manipal University, Manipal, "Triphibious Vehicle", Indian Patent Application No 201741027807, (2017). 